Rev. Biol. Neotrop. 5(2): 51-63, 2008

\title{
PREENSÃO DE ESPÉCIMES DA FAUNA SILVESTRE EM GOIÁS - SITUAÇÃO E DESTINAÇÃO
}

\author{
Lilian Freitas Bastos
}

Bióloga autônoma

\section{Vera Lúcia Ferreira Luz IsAíAs JosÉ dos ReIS}

Centro Nacional de Conservação e Manejo de Répteis e Anfíbios I (RAN/ICMBio), Rua 229 no 95, Setor Leste Universitário, 74.605-090, Goiânia, Goiás, Brasil

\section{Valéria Leão Souza}

Superintendência Municipal de Meio Ambiente, Rua Almiro de Moraes no 231, Centro, 75901-150, Rio Verde, Goiás, Brasil; e-mail: vleaosouza@gmail.com

\begin{abstract}
Resumo: Este estudo objetivou o levantamento das espécies silvestres encontradas com comerciantes ilegais e apreendidas no estado de Goiás, entre 1997 e 2005, visando identificar aquelas ameaçadas de extinção, quantificar os grupos mais apreendidos e conhecer a destinação dada aos espécimes. Foram analisados os registros obtidos a partir dos Termos de Apreensões e Depósitos (TADs), emitidos pela Superintendência do Instituto Brasileiro do Meio Ambiente e dos Recursos Naturais Renováveis (IBAMA)/ Batalhão de Polícia Militar Ambiental do estado de Goiás, e os dados são descritos em tabelas e figuras. Constatou-se a apreensão de 13.691 animais e 417,65 kg de produtos e/ou subprodutos da fauna silvestre. Do total de animais apreendidos, $94 \%$ eram aves, $4 \%$ mamíferos e $2 \%$ répteis, entre os quais, oito espécies constam na Lista das Espécies da Fauna Brasileira Ameaçadas de Extinção de 2003. Os principais locais de apreensão foram os municípios de Abadiânia, Alexânia, Aparecida de Goiânia, Aporé, Alvorada do Norte, Itumbiara, Sanclerlândia, Uruaçu e Goiânia. A maioria dos animais foi solta em áreas adequadas nos próprios municípios de apreensão e os demais foram destinados a zoológicos, centros de pesquisa e criadouros da região. Os resultados oferecem subsídios para o conhecimento sobre os crimes contra a fauna em Goiás, constituindo tema de discussão entre os órgãos envolvidos no planejamento de ações fiscalizatórias e educativas para o combate ao tráfico de espécies silvestres.
\end{abstract}

Palavras-chave: Apreensão, fauna silvestre, Goiás, tráfico.

Aвstract: The study aimed at evaluating the wild species found with illegal traders and thus apprehended in the state of Goiás, Brazil, between 1997 and 2005, in order to identify those considered endangered species, quantify the most common groups apprehended and know the destination given to the animals. We analyzed the records obtained in the Apprehension and Deposit Terms (TADs), emitted by the Superintendence of the Instituto Brasileiro do Meio Ambiente e dos Recursos Naturais Renováveis (IBAMA, Brazilian Institute of the Environment and Renewable Natural Resources)/Environmental Military Police Battalion of the state of Goiás and the data are presented in tables and graphs. We detected the apprehension of 13,691 animals and $417.65 \mathrm{~kg}$ of products and/or sub-products of the wild fauna. Among the total number of apprehended animals, $94 \%$ were birds, $4 \%$ mammals, and $2 \%$ reptiles and eight species are listed in the 2003 National List of Endangered Species of the Brazilian Fauna. The main places of apprehension were Abadiânia, Alexânia, Aparecida de Goiânia, Aporé, Alvorada do Norte, Itumbiara, Sanclerlândia, Uruaçu, and Goiânia. Most animals were released in suitable areas in the municipalities of apprehension and the others were sent to zoos, research centers, and regional legal breeding farms. The results provide data to get better knowledge of crimes against wildlife in Goiás and constitute important theme of discussion for the agencies that are involved in planning surveillance, patrolling, and educational actions to combat wild species trafficking.

KEY words: Apprehension, wildlife, Goiás, trafficking. 


\section{INTRODUÇÃO}

exuberância da fauna e flora
brasileira há muito tempo vem
despertando a cobiça mundial por sua rica e preciosa diversidade de espécies. Essa riqueza gerou a ideia de que a biodiversidade brasileira, além de abundante, era inesgotável e, por isso, vem sendo explorada de forma desordenada e predatória desde os tempos coloniais (Santos \& Câmara, 2002).

Apesar de ter sofrido esse tipo de agressão, o Brasil ainda é detentor da maior diversidade do planeta, contribuindo com aproximadamente $14 \%$ da biota mundial, o que o coloca entre os países dotados de megadiversidade (Costa et al., 2005), em um grupo constituído por 12 nações, as quais abrigam $70 \%$ da biodiversidade total do planeta (IBGE, 2004).

Entretanto, vem sendo observado um declínio das populações animais em decorrência da destruição de seus habitats, bem como pelo tráfico, que representa a terceira maior atividade ilícita no mundo e a segunda maior no Brasil (Santos \& Câmara, 2002). O país é tido como um dos principais fornecedores de flora e fauna para o mercado mundial, ocorrendo a retirada de aproximadamente $12 \mathrm{mi}$ lhões de animais silvestres anualmente da natureza para atender esta atividade, com taxas de mortalidade que podem chegar a $90 \%$, desde o momento da captura até o destino final. Em torno de $30 \%$ do produto deste mercado ilegal é exportado e o restante é comercializado internamente (WWF, 1995).

O crescimento demográfico humano também contribui para acelerar o ritmo da extinção, mas a pressão do aumento populacional não é o único fator responsável por ela (Fuccio et al., 2003).

No Brasil, a caça e o comércio predatório da fauna silvestre passaram a ser ilegais somente no ano de 1967, com a criação do Instituto Brasileiro de Desenvolvimento Florestal (IBDF) e a promulgação da Lei n 5.197 (Brasil, 1967), que regulamenta a proteção à fauna, pois até então não havia legislação que coibisse essas atividades (RENCTAS, 2001).

O comércio ilegal de animais silvestres está associado a problemas culturais e de educação, pobreza, falta de opções econômicas, desejo de lucro fácil e rápido, status e satisfação pessoal de manter animais silvestres como animais de estimação (RENCTAS, 2002).

A intensa retirada de exemplares da natureza configura sério problema para a conservação das espécies. Os principais consumidores dos produtos gerados por esse tráfico são, em ordem de importância: colecionadores particulares e zoológicos; interessados em biotecnologia (biopirataria), responsáveis pelo tráfico de aranhas, escorpiões, serpentes e inúmeras espécies de plantas; interessados em animais de estimação e comerciantes de produtos de fauna silvestre para serem utilizados como material para artesanato e peças de vestuário (Santos \& Câmara, 2002).

O tráfico de animais retira anualmente da natureza cerca de 38 milhões de indivíduos de diferentes grupos de organismos no planeta (RENCTAS, 2001). Estima-se que o comércio ilícito de vida silvestre, no qual se incluem a flora, a fauna e seus produtos e subprodutos, movimente anualmente de 10 a 20 bilhões de dólares por todo o mundo e que o Brasil participa com cerca de 5\% a 15\% deste total (Rocha, 1995).

Embora exista no Brasil um conjunto de leis para nortear as ações operacionais necessárias no combate ao tráfico de animais, observa-se a carência de uma política ou mesmo de um plano de ação explícito e direcionado para coibir este tipo de crime.

O Instituto Brasileiro do Meio Ambiente e dos Recursos Naturais Renováveis (IBAMA), dentro de suas atribuições de órgão fiscalizador, depara-se com sérios problemas no que se refere ao tráfico de animais silvestres. A vasta extensão do país, aliada à deficiência de recursos humanos e financeiros, entre outros fatores, são apenas algumas das dificuldades enfrentadas para agir de forma mais efetiva de modo a tentar coibir essa prática ilícita tão disseminada no cenário nacional. Atualmente, a fiscalização do IBAMA ganhou em qualidade com a utilização de novas tecnologias, como o sensoriamento remoto e o georreferenciamento.

Em Goiás, os animais silvestres apreendidos são centralizados na Superintendência do IBAMA sediada em Goiânia, onde o sistema de registro das apreensões é organizado. 
Apesar dos danos que o tráfico causa à fauna silvestre brasileira, há carência generalizada de informações quantitativas sobre o tema, o que dificulta a avaliação real da dimensão do problema e de seu impacto no Brasil (IBGE, 2004).

$\mathrm{O}$ intuito do presente estudo foi caracterizar as espécies da fauna silvestre apreendidas no estado de Goiás entre 1997 e 2005, visando identificar as espécies ameaçadas de extinção, quantificar os grupos mais apreendidos e conhecer a destinação dada aos espécimes.

\section{Material e Métodos}

Este estudo foi realizado a partir das informações registradas nos Termos de Apreensões e Depósitos (TADs) entre 1997 e 2005, emitidos pela Divisão de Fiscalização da Superintendência do IBAMA, juntamente com o Batalhão de Polícia Militar Ambiental do estado de Goiás, em Goiânia.

Foram analisados 2.830 registros, dos quais foram coletados os dados sobre o número do auto de infração, quando este foi gerado, a data em que os animais foram apreendidos, a identificação da espécie, o local de apreensão, a quantidade e o destino dos animais, seus produtos e/ou subprodutos.

Posteriormente, os dados foram ordenados por grupo animal para identificação das espécies, verificando se estas constavam na Lista das Espécies da Fauna Brasileira Ameaçadas de Extinção (Brasil, 2003), e organiza- dos de forma descritiva em tabelas e figuras construídas em planilha eletrônica (Microsoft Excel).

\section{Resultados e Discussão}

Entre 1997 e 2005, a Divisão de Fiscalização da Superintendência do IBAMA, isoladamente ou em ações eventuais com o Batalhão de Polícia Militar Ambiental do estado de Goiás, apreendeu 13.691 animais da fauna silvestre, pertencentes a três grupos: mamíferos, aves e répteis (Tabela 1). Além disso, também houve a apreensão de 417,65 $\mathrm{kg}$ de produtos e/ou subprodutos da fauna, entre os quais havia xenartros, crocodilianos, ungulados e artiodáctilos, em sua maioria.

A média anual de apreensão foi de 1.521 animais, tendo ocorrido o maior número de apreensões em 1999, com 2.891 espécimes, e o menor em 1997, com 109 exemplares (Figura 1). A grande discrepância entre os dados pode ser decorrente de flutuações, tanto nas quantidades traficadas quanto, principalmente, na intensidade e rigor da fiscalização de estradas, feiras e aeroportos que, de forma geral, carece de sistematização, planejamento adequado e recursos suficientes para o êxito das operações, como retratado pelo IBGE (2004).

Geralmente, o número de animais apreendidos é bem menor que aquele traficado, pois a quantidade de animais retirados da natureza é, muitas vezes, maior que a efetivamente comercializada em função das perdas

Tabela 1 - Animais silvestres apreendidos anualmente no estado de Goiás pelo IBAMA/Batalhão de Polícia Militar Ambiental do estado de Goiás, no período entre 1997 e 2005.

\begin{tabular}{ccccc}
\hline Ano & Répteis & Mamíferos & Aves & Total \\
\hline 1997 & 0 & 4 & 105 & 109 \\
1998 & 12 & 74 & 1.334 & 1.420 \\
1999 & 45 & 75 & 2.771 & 2.891 \\
2000 & 22 & 97 & 1.687 & 1.806 \\
2001 & 38 & 89 & 2.425 & 2.552 \\
2002 & 14 & 32 & 1.064 & 1.110 \\
2003 & 40 & 33 & 1.083 & 1.156 \\
2004 & 44 & 77 & 1.212 & 1.333 \\
2005 & 65 & 32 & 1.217 & 1.314 \\
\hline Total & 280 & 513 & 12.898 & 13.691 \\
\hline
\end{tabular}




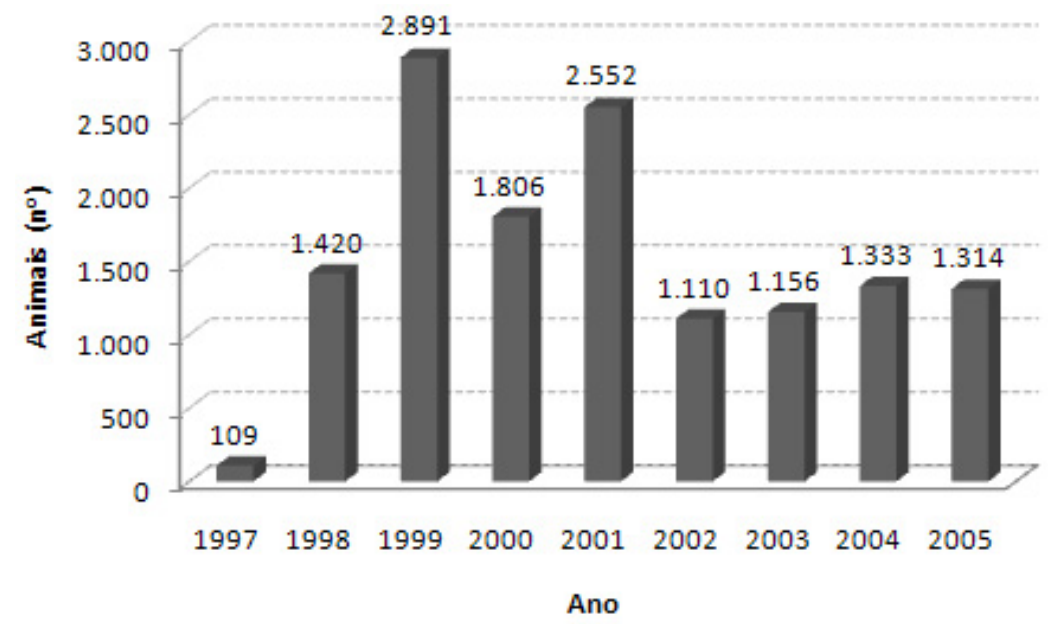

Figura 1 - Animais silvestres apreendidos anualmente no estado de Goiás pelo IBAMA/Batalhão de Polícia Militar Ambiental do estado de Goiás, no período entre 1997 e 2005.

que ocorrem durante o processo de captura e transporte. Estima-se que, para cada animal traficado, pelo menos três outros morram (IBGE, 2004).

Neste estudo, dos 13.691 animais apreendidos, aproximadamente $94 \%$ eram aves (principalmente, passeriformes e psitaciformes), 4\% mamíferos (artiodáctilos, edentados, primatas, ungulados e xenartros) e $2 \%$ répteis (crocodilianos, quelônios e serpentes) (Figura 2). Foram computadas 88 espécies de aves, 24 de mamíferos e 18 de répteis (Tabelas 2 a 4). A taxonomia dos grupos zoológicos seguiu as classificações propostas por Bérnils (2009), CBRO (2007) e Reis et al. (2006).
De acordo com os estudos realizados por Bezerra et al. (2004), Borges et al. (2004), Borges et al. (2006), Pereira \& Brito (2005), SEMA / PMA-SP (2006), bem como pelo resultado divulgado pela RENCTAS (2001) em âmbito nacional, a maior parte da fauna apreendida é composta por aves. Isso demonstra claramente a preferência do tráfico por esses animais que, muitas vezes, são destinados a colecionadores e pet shops. Entre as aves, as espécies mais almejadas pelo tráfico são os passeriformes, que além de atingir preço menor no mercado negro em relação aos outros animais, podem ser transportados clandestinamente em locais pequenos (SEMA/PMA-SP, 2006).

\section{Animais $\left(n^{\circ}\right)$}

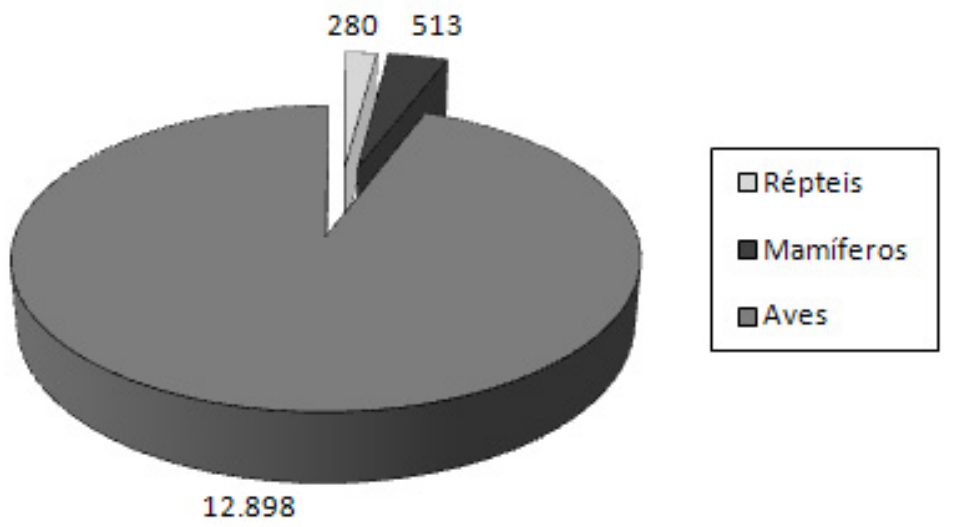

Figura 2 - Fauna silvestre apreendida no estado de Goiás pelo IBAMA/Batalhão de Polícia Militar Ambiental do estado de Goiás, de acordo com as classes, no período entre 1997 e 2005. 
Tabela 2 - Relação das espécies de aves apreendidas no estado de Goiás pelo IBAMA/Batalhão de Polícia Militar Ambiental do estado de Goiás, no período entre 1997 e 2005.

\begin{tabular}{|c|c|c|}
\hline Táxon & Nome comum & $\begin{array}{c}\text { Apreensões } \\
\left(n^{\circ}\right)\end{array}$ \\
\hline \multicolumn{3}{|l|}{ STRUTHIONIFORMES } \\
\hline \multicolumn{3}{|l|}{ RHEIDAE } \\
\hline Rhea americana (Linnaeus, 1758) & Ema & 56 \\
\hline \multicolumn{3}{|l|}{ TINAMIFORMES } \\
\hline \multicolumn{3}{|l|}{ TINAMIDAE } \\
\hline Crypturellus noctivagus (Wied, 1820) & Jaó-do-sul & 6 \\
\hline Crypturellus undulatus (Temminck, 1815) & Jaó & 6 \\
\hline Nothura maculosa (Temminck, 1815) & Codorna-amarela & 1 \\
\hline Rhynchotus rufescens (Temminck, 1815) & Perdiz & 23 \\
\hline \multicolumn{3}{|l|}{ ANSERIFORMES } \\
\hline \multicolumn{3}{|l|}{ ANATIDAE } \\
\hline Cairina moschata (Linnaeus, 1758) & Pato-do-mato & 2 \\
\hline \multicolumn{3}{|l|}{ GALLIFORMES } \\
\hline \multicolumn{3}{|l|}{ CRACIDAE } \\
\hline Crax fasciolata (Spix, 1825) & Mutum-de-penacho & 4 \\
\hline
\end{tabular}

CICONIIDAE

Jabiru mycteria (Lichtenstein, 1819)

Tuiuiú

5

FALCONIFORMES

ACCIPITRIDAE

Rupornis magnirostris (Gmelin, 1788)

Gavião-carijó

2

FALCONIDAE

Caracara plancus (Miller, 1777)

Caracará

1

\begin{tabular}{|c|c|c|}
\hline \multicolumn{3}{|l|}{ GRUIFORMES } \\
\hline \multicolumn{3}{|l|}{ RALLIDAE } \\
\hline Pardirallus maculatus (Boddaert, 1783) & Saracura-carijó & 7 \\
\hline \multicolumn{3}{|l|}{ CARIAMIDAE } \\
\hline Cariama cristata (Linnaeus, 1766) & Seriema & 4 \\
\hline \multicolumn{3}{|l|}{ COLumbiformes } \\
\hline \multicolumn{3}{|l|}{ ColumbiDae } \\
\hline Columbina passerina (Linnaeus, 1758) & Rolinha-cinzenta & 3 \\
\hline Columbina squammata (Lesson, 1831) & Fogo-apagou & 7 \\
\hline Columbina talpacoti (Temminck, 1811) & Rolinha-roxa & 60 \\
\hline Leptotila rufaxilla (Richard \& Bernard, 1792) & Juriti-gemedeira & 8 \\
\hline Zenaida auriculata (Des Murs, 1847) & Pomba-de-bando & 45 \\
\hline \multicolumn{3}{|l|}{ PSITTACIFORMES } \\
\hline \multicolumn{3}{|l|}{ PsitTAcidae } \\
\hline Alipiopsitta xanthops (Spix, 1824) & Papagaio-galego & 32 \\
\hline Amazona aestiva (Linnaeus, 1758) & Papagaio-verdadeiro & 62 \\
\hline Amazona amazonica (Linnaeus, 1766) & Curica & 4 \\
\hline Amazona brasiliensis (Linnaeus, 1758) & Papagaio-de-cara-roxa & 1 \\
\hline Amazona pretrei (Temminck, 1830) & Papagaio-charão & 1 \\
\hline
\end{tabular}


Continuação

Tabela 2 - Relação das espécies de aves apreendidas no estado de Goiás pelo IBAMA/Batalhão de Polícia Militar Ambiental do estado de Goiás, no período entre 1997 e 2005.

\begin{tabular}{|c|c|c|}
\hline Táxon & Nome comum & $\begin{array}{c}\text { Apreensões } \\
\left(n^{\circ}\right)\end{array}$ \\
\hline Amazona sp. & Papagaio & 548 \\
\hline Anodorynchus hyacinthinus (Latham, 1790) & Arara-azul-grande & 36 \\
\hline Ara ararauna (Linnaeus, 1758) & Arara-canindé & 47 \\
\hline Ara chloropterus (Gray, 1859) & Arara-vermelha-grande & 20 \\
\hline Ara severus (Linnaeus, 1758) & Maracanã-guaçu & 2 \\
\hline Ara sp. & Arara & 37 \\
\hline Aratinga aurea (Gmelin, 1788) & Periquito-rei & 1 \\
\hline Aratinga jandaya (Gmelin, 1788) & Jandaia-verdadeira & 15 \\
\hline Aratinga sp. & Periquito & 237 \\
\hline Brotogeris chiriri (Vieillot, 1818) & Periquito-de-encontro-amarelo & 17 \\
\hline Brotogeris tirica (Gmelin, 1788) & Periquito-rico & 33 \\
\hline Diopsittaca nobilis (Linnaeus, 1758) & Maracanã-pequena & 76 \\
\hline Forpus xanthopterygius (Spix, 1824) & Tuim & 2 \\
\hline Graydidascalus brachyurus (Kuhl, 1820) & Curica-verde & 4 \\
\hline Pionites leucogaster (Kuhl, 1820) & Marianinha-de-cabeça-amarela & 1 \\
\hline Primolius maracana (Vieillot, 1816) & Maracanã-verdadeira & 2 \\
\hline \multicolumn{3}{|l|}{ APODIFORMES } \\
\hline \multicolumn{3}{|l|}{ TROCHILIDAE } \\
\hline Calliphlox amethystina (Boddaert, 1783) & Estrelinha-ametista & 6 \\
\hline Lophornis magnificus (Vieillot, 1817) & Topetinho-vermelho & 1 \\
\hline \multicolumn{3}{|l|}{ Piciformes } \\
\hline \multicolumn{3}{|l|}{ RHAMPHASTIDAE } \\
\hline Ramphastos toco (Statius Muller, 1776) & Tucanuçu & 18 \\
\hline \multicolumn{3}{|l|}{ PICIDAE } \\
\hline Melanerpes candidus (Otto, 1796) & Birro, Pica-pau-branco & 2 \\
\hline \multicolumn{3}{|l|}{ PASSERIFORMES } \\
\hline \multicolumn{3}{|l|}{ CONOPOPHAGIDAE } \\
\hline Conopophaga lineata (Wied, 1831) & Chupa-dente & 18 \\
\hline \multicolumn{3}{|l|}{ CotINGIDAE } \\
\hline Procnias nudicollis (Vieillot, 1817) & Araponga & 5 \\
\hline \multicolumn{3}{|l|}{ CORVIDAE } \\
\hline Cyanocorax sp. & Gralha & 3 \\
\hline Pica pica (Linnaeus, 1758) & Pega & 12 \\
\hline \multicolumn{3}{|l|}{ Troglodytidae } \\
\hline Cistothorus platensis (Latham, 1790) & Corruíra-do-campo & 2 \\
\hline Troglodytes musculus (Naumann, 1823) & Corruíra & 12 \\
\hline \multicolumn{3}{|l|}{ TURDIDAE } \\
\hline Turdus amaurochalinus (Cabanis, 1850) & Sabiá-poca & 1 \\
\hline Turdus fumigatus (Lichtenstein, 1823) & Sabiá-da-mata & 3 \\
\hline Turdus rufiventris (Vieillot, 1818) & Sabiá-laranjeira & 16 \\
\hline Turdus sp. & Sabiá & 205 \\
\hline \multicolumn{3}{|l|}{ COEREBIDAE } \\
\hline Coereba flaveola (Linnaeus, 1758) & Cambacica & 1 \\
\hline
\end{tabular}


Conclusão

Tabela 2 - Relação das espécies de aves apreendidas no estado de Goiás pelo IBAMA/Batalhão de Polícia Militar Ambiental do estado de Goiás, no período entre 1997 e 2005.

\begin{tabular}{|c|c|c|}
\hline Táxon & Nome comum & $\begin{array}{c}\text { Apreensões } \\
\left(n^{\circ}\right)\end{array}$ \\
\hline \multicolumn{3}{|l|}{ THRAUPIDAE } \\
\hline Ramphocelus bresilius (Linnaeus, 1766) & Tiê-sangue & 14 \\
\hline Tangara sp. & Saíra & 10 \\
\hline Thraupis episcopus (Linnaeus, 1766) & Sanhaçu-da-amazônia & 4 \\
\hline Thraupis sayaca (Linneus, 1766) & Sanhaçu-cinzento & 31 \\
\hline \multicolumn{3}{|l|}{ EMBERIZIDAE } \\
\hline Amaurospiza moesta (Hartlaub, 1853) & Negrinho-do-mato & 1 \\
\hline Coryphospingus cucullatus (Statius Muller, 1776) & Tico-tico-rei & 4 \\
\hline Paroaria dominicana (Linnaeus, 1758) & Cardeal-do-nordeste & 171 \\
\hline Paroaria capitata (d'Orbigny \& Lafresnaye, 1837) & Cavalaria & 1 \\
\hline Sporophila caerulescens (Vieillot, 1817) & Coleirinho & 143 \\
\hline Volatinia jacarina (Linnaeus, 1766) & Tiziu & 8 \\
\hline Sicalis columbiana (Cabanis, 1851) & Canário-do-amazonas & 2 \\
\hline Sicalis flaveola (Linnaeus, 1766) & Canário-da-terra-verdadeiro & 6.932 \\
\hline Sicalis sp. & Canário & 2 \\
\hline Sporophila angolensis (Linnaeus, 1766) & Curió & 1.836 \\
\hline Sporophila collaris (Boddaert, 1783) & Coleiro-do-brejo & 28 \\
\hline Sporophila lineola (Linnaeus, 1758) & Bigodinho & 59 \\
\hline Sporophila maximiliani (Cabanis, 1851) & Bicudo & 29 \\
\hline Sporophila melanogaster (Pelzeln, 1870) & Caboclinho-de-barriga-preta & 33 \\
\hline Sporophila nigricollis (Vieillot, 1823) & Baiano & 526 \\
\hline Sporophila plumbea (Wied, 1830) & Patativa & 80 \\
\hline Zonotrichia capensis (Statius Muller, 1776) & Tico-tico & 13 \\
\hline \multicolumn{3}{|l|}{ CARDINALIDAE } \\
\hline Cyanocompsa brissonii (Lichtenstein, 1823) & Azulão & 119 \\
\hline Saltator fuliginosus (Daudin, 1800) & Pimentão & 3 \\
\hline Saltator similis (d’Orbigny \& Lafresnaye, 1837) & Trinca-ferro-verdadeiro & 67 \\
\hline \multicolumn{3}{|l|}{ PARULIDAE } \\
\hline Basileuterus culicivorus (Deppe, 1830) & Pula-pula & 5 \\
\hline \multicolumn{3}{|l|}{ ICTERIDAE } \\
\hline Cacicus cela (Linnaeus, 1758) & Xexéu & 1 \\
\hline Cacicus sp. & Guaxe & 54 \\
\hline Gnorimopsar chopi (Vieillot, 1819) & Graúna & 882 \\
\hline Icterus icterus (Linnaeus, 1766) & Sofrê & 18 \\
\hline Scaphidura oryzivora (Gmelin, 1788) & Graúna & 1 \\
\hline \multicolumn{3}{|l|}{ FrINGILLIDAE } \\
\hline Carduelis magellanica (Vieillot, 1805) & Pintassilgo & 83 \\
\hline Euphonia chlorotica (Linnaeus, 1766) & Fim-fim & 5 \\
\hline Euphonia violacea (Linnaeus, 1758) & Gaturamo-verdadeiro & 9 \\
\hline \multicolumn{3}{|l|}{ ESTRILDIDAE } \\
\hline Estrilda astrild (Linnaeus, 1758) & Bico-de-lacre & 1 \\
\hline \multicolumn{3}{|l|}{ PASSERIDAE } \\
\hline Passer domesticus (Linnaeus, 1758) & Pardal & 1 \\
\hline
\end{tabular}


Tabela 3 - Relação das espécies de mamíferos apreendidas no estado de Goiás pelo IBAMA/Batalhão de Polícia Militar Ambiental do estado de Goiás Goiás, no período entre 1997 e 2005.

\begin{tabular}{|c|c|c|}
\hline Táxon & Nome comum & Apreensões $\left(\mathrm{n}^{\circ}\right)$ \\
\hline \multicolumn{3}{|l|}{ XENARTHRA } \\
\hline \multicolumn{3}{|l|}{ DASYPODIDAE } \\
\hline Dasypus novemcinctus (Linnaeus, 1758) & Tatu-galinha & 11 \\
\hline Dasypus septemcinctus (Linnaeus, 1758) & Tatu-de-sete-cintas & 21 \\
\hline Dasypus sp. & Tatu & 163 \\
\hline Euphractus sexcinctus (Linnaeus, 1758) & Tatu-peba & 18 \\
\hline \multicolumn{3}{|l|}{ EDENTATA } \\
\hline \multicolumn{3}{|l|}{ MYRMECOPHAGIDAE } \\
\hline Myrmecophaga tridactyla (Linnaeus, 1758) & Tamanduá-bandeira & 1 \\
\hline Tamandua tetradactyla (Linnaeus, 1758) & Tamanduá-mirim & 3 \\
\hline \multicolumn{3}{|l|}{ Primates } \\
\hline \multicolumn{3}{|l|}{ CALLITRICHIDAE } \\
\hline Callithrix penicillata (Geoffroy, 1812) & Mico-estrela & 8 \\
\hline Saguinus imperator (Goeldi, 1907) & Sagui-de-bigode & 1 \\
\hline \multicolumn{3}{|l|}{ CEBIDAE } \\
\hline Cebus apella (Kuhl, 1820) & Macaco-prego & 42 \\
\hline \multicolumn{3}{|l|}{ CARNIVORA } \\
\hline \multicolumn{3}{|l|}{ CANIDAE } \\
\hline Cerdocyon thous (Linnaeus, 1766) & Cachorro-do-mato & 1 \\
\hline Chrysocyon brachyurus (Illiger, 1815) & Lobo-guará & 1 \\
\hline \multicolumn{3}{|l|}{ FELIDAE } \\
\hline Leopardus pardalis (Linnaeus, 1758) & Jaguatirica & 4 \\
\hline Leopardus tigrinus (Schreber, 1775) & Gato-do-mato-pequeno & 1 \\
\hline \multicolumn{3}{|l|}{ Procyonidae } \\
\hline Nasua nasua (Linnaeus, 1766) & Quati & 10 \\
\hline \multicolumn{3}{|l|}{ PerISSODACTYLa } \\
\hline \multicolumn{3}{|l|}{ TAPIRIDAE } \\
\hline Tapirus terrestris (Linnaeus, 1758) & Anta & 2 \\
\hline \multicolumn{3}{|l|}{ ARTIODACTYLA } \\
\hline \multicolumn{3}{|l|}{ TAYASSUIDAE } \\
\hline Pecari tajacu (Linnaeus, 1758) & Cateto & 76 \\
\hline Tayassu pecari (Link, 1795) & Queixada & 14 \\
\hline \multicolumn{3}{|l|}{ CERVIDAe } \\
\hline Mazama americana (Erxleben, 1777) & Veado-mateiro & 2 \\
\hline Mazama gouazoupira (G. Fischer, 1814) & Veado-catingueiro & 15 \\
\hline Ozotoceros bezoarticus (Linnaeus, 1758) & Veado-campeiro & 2 \\
\hline Ozotocerus sp. & Veado & 18 \\
\hline \multicolumn{3}{|l|}{ RODENTIA } \\
\hline \multicolumn{3}{|l|}{ HYDROCHAERIDAE } \\
\hline Hydrochaeris hydrochaeris (Linnaeus, 1766) & Capivara & 46 \\
\hline \multicolumn{3}{|l|}{ DASYPROCTIDAE } \\
\hline Agouti paca (Linnaeus, 1766) & Paca & 32 \\
\hline Dasyprocta agouti (Linnaeus, 1766) & Cutia & 21 \\
\hline
\end{tabular}


Tabela 4 - Relação das espécies de répteis apreendidas no estado de Goiás pelo IBAMA/Batalhão de Polícia Militar Ambiental do estado de Goiás Goiás, no período entre 1997 e 2005.

\begin{tabular}{|c|c|c|}
\hline Táxon & Nome comum & Apreensões $\left(n^{\circ}\right)$ \\
\hline \multicolumn{3}{|l|}{ Testudines } \\
\hline \multicolumn{3}{|l|}{ EMYDIDAE } \\
\hline Trachemys dorbigni (Duméril \& Bibron, 1835) & Tigre-d'água & 1 \\
\hline \multicolumn{3}{|l|}{ TESTUDINIDAE } \\
\hline Chelonoidis sp. & Jabuti & 131 \\
\hline \multicolumn{3}{|l|}{ PODOCNEMIDIDAE } \\
\hline Podocnemis expansa (Schweigger, 1812) & Tartaruga-da-amazônia & 73 \\
\hline \multicolumn{3}{|l|}{ CHELIDAe } \\
\hline Phrynops sp. & Cágado & 11 \\
\hline \multicolumn{3}{|l|}{ CROCODYLIA } \\
\hline \multicolumn{3}{|l|}{ AlLIGATORIDAE } \\
\hline Caiman sp. & Jacaré & 36 \\
\hline Paleosuchus palpebrosus (Cuvier, 1807) & Jacaré-coroa & 4 \\
\hline \multicolumn{3}{|l|}{ SQUAMATA } \\
\hline \multicolumn{3}{|l|}{ IgUANIDAE } \\
\hline Iguana iguana (Linnaeus, 1758) & Iguana & 1 \\
\hline \multicolumn{3}{|l|}{ BOIDAE } \\
\hline Boa constrictor (Linnaeus, 1758) & Jibóia & 2 \\
\hline Boa constrictor constrictor (Forcart, 1960) & Jibóia-da-amazônia & 1 \\
\hline Corallus hortulanus (Linnaeus, 1758) & Jararaca-do-oco & 3 \\
\hline \multicolumn{3}{|l|}{ COlubridae } \\
\hline Helicops modestus (Günter, 1861) & Cobra-d'água & 2 \\
\hline Liophis sp. & Cobra-capinzeira & 1 \\
\hline Oxyrhopus trigeminus (Duméril, Bibron \& Duméril, 1854) & Coral-falsa & 6 \\
\hline Philodryas olfersii (Lichtenstein, 1823) & Cobra-cipó & 1 \\
\hline Sibynomorphus mikanii (Schlegel, 1837) & Dormideira & 1 \\
\hline \multicolumn{3}{|l|}{ ELAPIDAE } \\
\hline Micrurus sp. & Coral & 1 \\
\hline \multicolumn{3}{|l|}{ VIPERIDAE } \\
\hline Bothrops sp. & Jararaca & 2 \\
\hline Crotalus durissus (Linnaeus, 1758) & Cascavel & 3 \\
\hline
\end{tabular}

Além do animal vivo, um indeterminado número de aves, répteis e mamíferos é sacrificado e suas penas, couros e outras partes são comercializados a cada ano, assim como os ovos.

Em seu estudo com universitários, Piraiano \& Molina (2001) constataram que as aves ficaram em segundo lugar na preferência dos estudantes como animais de estimação. Paradoxalmente, o mesmo estudo demonstrou que os graduandos de Ciências Biológicas eram os que mais possuíam animais silvestres em suas residências. Isso mostra, sem dúvida alguma, a urgência de se estabelecer uma cadeira de ética (ausente na maioria das universidades) para este curso, além de seminários e debates com o intuito de informar sobre os problemas e mazelas do tráfico de animais silvestres (Borges et al., 2006).

Entre os animais apreendidos pelo IBAMA/Batalhão de Polícia Militar Ambiental, oito espécies (Tabela 5) constavam na Lista das Espécies da Fauna Brasileira Ameaça- 


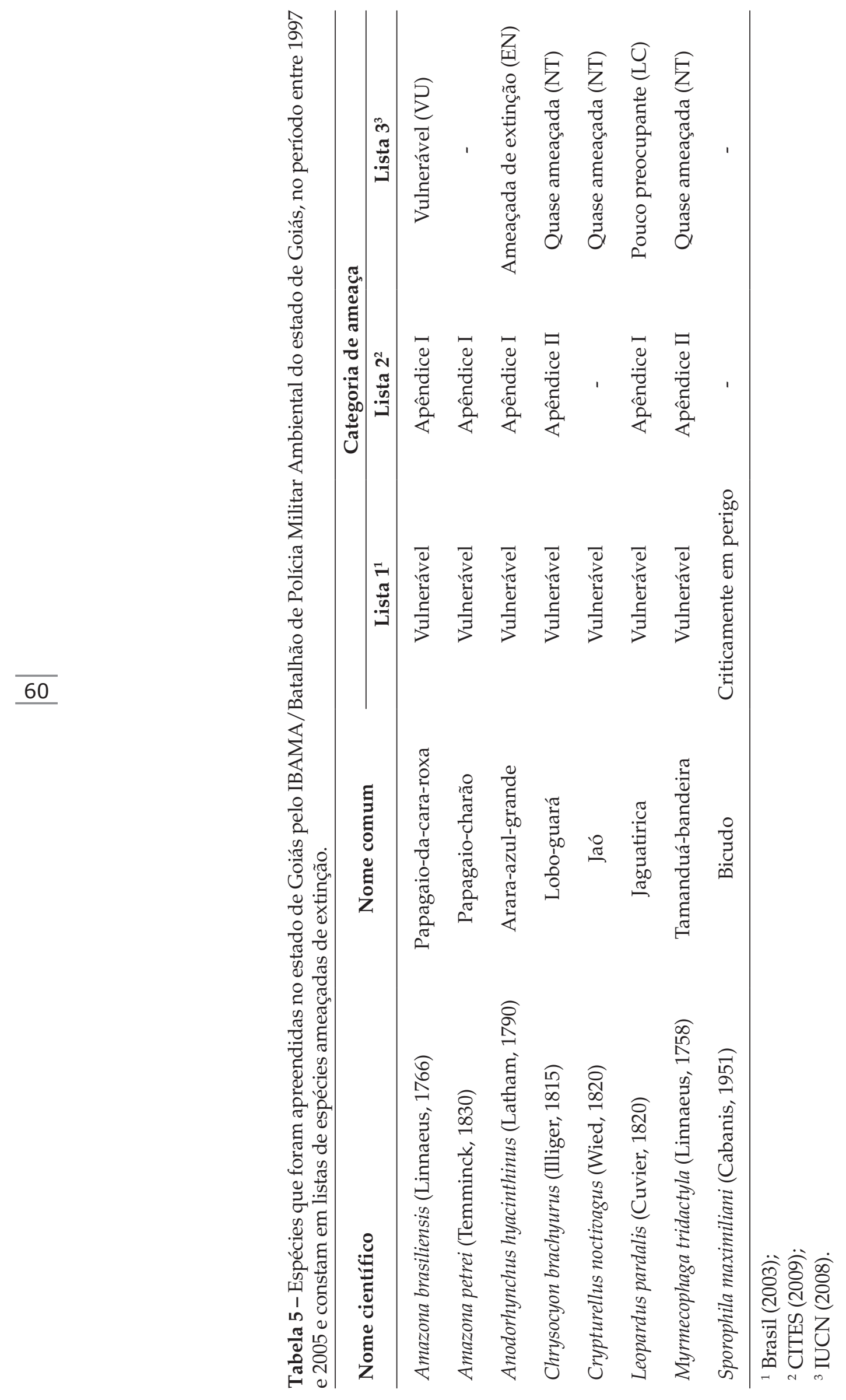


das de Extinção de 2003 (Brasil, 2003), entre as quais, três espécies (Anodorhynchus hyacinthinus Latham, 1790 - arara-azul-grande, Oryzoborus maximiliani Cabanis, 1951 - bicudo e Chrysocyon brachyurus Illiger, 1815 - lobo-guará) também foram relatadas por Borges et al. (2006) na região de Juiz de Fora, Minas Gerais.

A arara-azul-grande (Anodorhynchus hyacinthinus Latham, 1790), o papagaio-de-cararoxa (Amazona brasiliensis Linnaeus, 1766) e a jaguatirica (Leopardus pardalis Linnaeus, 1758) estão entre as espécies mais visadas por colecionadores particulares e zoológicos por sua raridade e maior valor de mercado (RENCTAS, 2001).

Os produtos da fauna silvestre são muito utilizados para fabricar adornos e artesanato. As espécies envolvidas variam ao longo dos tempos, de acordo com os costumes e os mercados da moda. Normalmente, são comercializados couros, peles, penas, garras, presas, além de diversas outras partes de animais. Todos esses produtos entram no mercado de moda e suvenirs para turistas (RENCTAS, 2001).

Neste estudo, foram apreendidos produtos e/ou subprodutos de 13 espécies da fauna silvestre (Tabela 6).
Os registros das operações de fiscalização dos órgãos envolvidos foram procedentes de 193 municípios do estado de Goiás. Entre esses, os principais locais de apreensão da fauna silvestre ocorreram nos municípios de Abadiânia, Alexânia, Aparecida de Goiânia, Aporé, Alvorada do Norte, Itumbiara, Sanclerlândia, Uruaçu e Goiânia.

A maioria dos animais foi solta em áreas próximas aos municípios onde foram apreendidos, enquanto os outros foram destinados a zoológicos, centros de pesquisa e triagem e criadouros comerciais e conservacionistas da região. Os produtos e/ou subprodutos apreendidos foram doados a instituições filantrópicas. Informações incompletas registradas nos TADs não deixaram explícita a quantidade de animais apreendidos que tiveram essas destinações.

No levantamento realizado pela RENCTAS (2001) constatou-se que a maior parte da fauna apreendida é solta. Essa soltura é prevista pela Lei $n^{\circ} 9.605$ (Brasil, 1998) e ratificada pelo Decreto n ${ }^{\circ} 6.514$ (Brasil, 2008), embora deva ser vinculada a programas específicos de manejo das espécies, os quais devem ser aprovados pelo IBAMA. Ainda segundo o relatório RENCTAS (2001), na maioria das vezes, ocorre

Tabela 6 - Espécies da fauna silvestre cujos produtos e/ou subprodutos foram apreendidos no estado de Goiás pelo IBAMA/Batalhão de Polícia Militar Ambiental do estado de Goiás, no período entre 1997 e 2005.

\begin{tabular}{lccc}
\hline Nome científico & Nome comum & Produto/ subproduto & Quantidade \\
\hline Agouti paca (Linnaeus, 1766) & & & $(\mathrm{kg})$ \\
Caiman sp. & Paca & Carne & 21,0 \\
Crypturellus undulatus (Temminck, 1815) & Jacaré & Carne & 134,4 \\
Dasyprocta agouti (Linnaeus, 1766) & Jaó & Carne & 0,8 \\
Dasypus septemcinctus (Linnaeus, 1758) & Cutia & Carne & 1,0 \\
Dasypus sp. & Tatu-de-sete-cintas & Carne, carapaça & 1,0 \\
Euphractus sexcinctus (Linnaeus, 1758) & Tatu & Carne, carapaça & 2,0 \\
Hydrochaeris hydrochaeris (Linnaeus, 1766) & Tatu-peba & Carne & 1,0 \\
Ozotocerus bezoarticus (Linnaeus, 1758) & Capivara & Carne & 149,0 \\
Ozotoceros sp. & Veado-campeiro & Carne & 4,0 \\
Pecari tajacu (Linnaeus, 1758) & Veado & Carne, cabeça & 12,0 \\
Podocnemis expansa (Schweigger, 1812) & Cateto & Carne & 45,4 \\
Tartaruga-da-amazônia & & & \\
Tapirus terrestris (Linnaeus, 1758) & Carne, carapaça & 6,0 & \\
\hline & Anta & Carne & 40,0 \\
\hline
\end{tabular}


soltura sem critério científico algum, apenas liberando os animais no próprio local de apreensão. Isso acontece por falta de informação dos órgãos fiscalizadores e por falta de Centros de Triagem de Animais Silvestres (CETAS) capacitados para receber os animais apreendidos.

Em Goiás, apesar da implantação de um CETAS em 2007, no município de Goiânia, ainda há carência para atender a demanda de recepção e manutenção dos animais e permitir análise mais criteriosa da fauna em apoio à atividade de fiscalização.

A destinação adequada da fauna silvestre apreendida apresenta-se como um dos principais problemas a serem resolvidos pelos órgãos ambientais (Vidolin et al., 2004). Além disso, as atitudes e cuidados governamentais quanto ao uso e à destinação da fauna apreendida devem ser avaliados de forma técnica, legal e ética, sendo obrigação das autoridades constituídas proporcionar destino apropriado aos animais apreendidos, viabilizando a implantação de locais adequadamente constituídos e equipados, onde sejam efetuadas a identificação e a triagem dos exemplares, além da reabilitação ou da destinação para programas de criação e manutenção em cativeiro.

Este estudo oferece subsídios para o conhecimento sobre os crimes contra a fauna ocorridos em Goiás, constituindo tema de discussão entre os órgãos envolvidos no planejamento de ações fiscalizatórias e educativas para o combate ao tráfico de animais silvestres.

\section{Agradecimentos}

Agradecemos ao Núcleo de Fauna e Recursos Pesqueiros (NUFARP) e à Divisão de Controle da Fiscalização (DICOF) da Superintendência do IBAMA/GO pela disponibilização dos dados utilizados no presente estudo.

\section{REFERÊNCIAS}

Bérnils, R. S. (Org.). 2009. Brazilian reptiles list of species. Curitiba, Sociedade Brasileira de Herpetologia. Disponível em: <http:/ / www.sbherpetologia.org.br/ checklist/repteis.htm>.

Bezerra, A. R. G. F., R. C. Costa, F. Lins \& M. L. P. Silva. 2004. Tráfico de animais silvestres: (II) Variação anual de espécies rece- bidas no Centro de Triagem de Animais Silvestres. In: $25^{\circ}$ Congresso Brasileiro de Zoologia, Brasília, DF, Universidade de Brasília. Resumos. Curitiba, Sociedade Brasileira de Zoologia, p. 473.

Borges, R. C., A. de Oliveira, N. Bernardo \& R. M. M. C. da Costa. 2006. Diagnóstico da fauna silvestre apreendida e recolhida pela Polícia Militar de Meio Ambiente de Juiz de Fora, MG (1998 e 1999). Rev. Bras. Zoociênc. 8: 23-33.

Borges, R. C., M. G. Silva, E. L. Bisaggio \& A. E. R. Nascimento. 2004. Levantamento das espécies e determinação da taxa de mortalidade da fauna apreendida/recolhida no Ibama/JF. In: $25^{\circ}$ Congresso Brasileiro de Zoologia, Brasília, DF, Universidade de Brasília. Resumos. Curitiba, Sociedade Brasileira de Zoologia, p. 476.

Brasil. 1967. Lei $n^{\circ} 5.197$, de 3 de janeiro de 1967. Dispõe sobre a proteção à fauna e dá outras providências. Diário Oficial [da] República Federativa do Brasil, Brasília, DF, 3 jan. 1967. Disponível em: <http:/ / www.planalto.gov.br/ccivil_03/Leis / L5197.htm>.

Brasil. 1998. Lei $\mathrm{n}^{\circ} 9.605$, de 12 de fevereiro de 1998. Dispõe sobre as sanções penais e administrativas derivadas de condutas e atividades lesivas ao meio ambiente, e dá outras providências. Diário Oficial [da] República Federativa do Brasil, Brasília, DF, 12 fev. 1998. Disponível em: <http:/ / www.planalto.gov.br/ccivil_03/LEIS/ L9605.htm>.

Brasil. 2003. Instrução Normativa MMA $\mathrm{n}^{\mathrm{o}} 3$, de 27 de maio de 2003. Ministério do Meio Ambiente, Brasília, DF, 22 p. Disponível em: <http://www. mpes.gov.br/anexos/centros_apoio/ arquivos/10_2093144032172008_ INSTRU $\%$ C3\%87\%C3\%83O\%20NORMATIVA \%20MMA\%20N\%C2\%B0\%20 3,\%20DE\%2027\%20DE\%20MAIO\%20 DE\%202003.pdf>.

Brasil. 2008. Decreto ${ }^{\circ}$ 6.514, de 22 de julho de 2008. Dispõe sobre as infrações e sanções administrativas ao meio ambiente, estabelece o processo administrativo federal para apuração destas infrações, e dá outras 
providências. Diário Oficial [da] República Federativa do Brasil, Brasília, DF, 22 jul. 2008. Disponível em: <http://www. planalto.gov.br / CCIVIL/_Ato20072010/2008/Decreto/D6514.htm>.

CBRO. Comitê Brasileiro de Registros Ornitológicos. 2007. Lista das aves do Brasil. $6^{a}$ ed. Rio de Janeiro. Disponível em: $<$ http:/ / www.cbro.org.br/CBRO/listabr. htm>.

CITES. Convention on International Trade in Endangered Species of Wild Fauna and Flora. 2009. CITES species database. Geneva. Disponível em: <http://www. cites.org/eng/resources/species.html>.

Costa, L. P., Y. R. L. Leite, S. L. Mendes \& A. D. Ditchfield. 2005. Conservação de mamíferos no Brasil. Megadiversidade 1: 103-112.

Fuccio, H., E. F. de Carvalho \& G. Vargas. 2003. Perfil da caça e dos caçadores no Estado do Acre, Brasil. Rev. Aportes Andinos 6: 1-18. Movimientos Sociales, Políticas de Seguridad y Democracia. Disponível em: <http:/ / www.uasb.edu.ec/padh/centro/pdfs6/Heloisa\%20Fuccio.pdf>.

IBGE. Instituto Brasileiro de Geografia e Estatística. 2004. Dimensão ambiental - biodiversidade. In: Indicadores de desenvolvimento sustentável-Brasil 2004. Brasília, DF, p. 100-134. Disponível em: <http:// www.institutohorus.org.br/download/ artigos/ibge_biodiversidade.PDF>.

IUCN. International Union for Conservation of Nature. 2008. IUCN Red List. Gland. Disponível em: <http://www.iucn.org/ about/work/programmes/species/red_ list $/>$.

Pereira, G. A. \& M. T. de Brito. 2005. Diversidade de aves silvestres brasileiras comercializadas nas feiras livres da Região Metropolitana do Recife, Pernambuco. Atual. Ornitol. 126: 14.
Piraiano, D. A. \& F. B. Molina. 2001. Estudantes universitários e animais de estimação: influência do curso/sexo na posse dos animais. In: $19^{\circ}$ Congresso Brasileiro de Etologia, Universidade Federal de Juiz de Fora, Juiz de Fora. Resumos. São Paulo, Sociedade Brasileira de Etologia, p. 314.

Reis, N. R., A. L. Peracchi, W. A. Pedro \& I. P. Lima. 2006. Mamíferos do Brasil. Nélio R. dos Reis, Londrina, 437 p. Disponível em: <http:/ /www.cienciamao.if.usp.br/ dados/pdf/_mamiferosdobrasilneliord. arquivopdf.pdf $>$.

RENCTAS. Rede Nacional de Combate ao Tráfico de Animais. 2001. $1^{\circ}$ Relatório nacional sobre o tráfico de animais silvestres. 107 p.

RENCTAS. Rede Nacional de Combate ao Tráfico de Animais. 2002. Animais silvestres: vida à venda. Dupligráfica, Brasília, DF, $260 \mathrm{p}$.

Rocha, F. M. 1995. Tráfico de animais silvestres no Brasil. WWF, Brasília, DF, 27 p.

Santos, T. C. C. \& J. B. D. Câmara (Orgs.). 2002. GeoBrasil 2002: perspectivas do meio ambiente no Brasil - o estado da biodiversidade. Edições IBAMA, Brasília, DF, 447 p.

SEMA/PMA-SP. Secretaria Estadual do Meio Ambiente/Polícia Militar Ambiental do estado de São Paulo. 2006. Tráfico de animais da fauna silvestre nacional. Dados estatísticos e estratégias operacionais 2001-2005. São Paulo, 26 p. Disponível em: <http:/ / www.pea.org.br/educativo/relatorio_ policia_ambiental.pdf $>$.

Vidolin, G. P., P. R. Mangini, M. de MouraBrito \& M. C. Muchailh. 2004. Programa estadual de manejo de fauna silvestre apreendida - Estado do Paraná, Brasil. Cad. Biodivers. 4: 37-49.

WWF. World Wildlife Foundation. 1995. Tráfico de animais silvestres no Brasil. $1^{\circ}$ Informe. Brasília, DF, 48 p. 